\title{
Simplified risk stratification criteria for identification of patients with MRSA bacteremia at low risk of infective endocarditis: implications for avoiding routine transesophageal echocardiography in MRSA bacteremia
}

\author{
P. Buitron de la Vega ${ }^{6,7}$ - P. Tandon ${ }^{3}$ - W. Qureshi ${ }^{8}$ - Y. Nasr ${ }^{5}$ • R. Jayaprakash ${ }^{4}$ • \\ S. Arshad ${ }^{1}$ - D. Moreno ${ }^{1}$ - G. Jacobsen ${ }^{9}$ K. Ananthasubramaniam ${ }^{2,5}$ • M. Ramesh ${ }^{1,2}$. \\ M. Zervos ${ }^{1,2}$
}

Received: 23 September 2015 / Accepted: 24 November 2015 / Published online: 16 December 2015

(C) The Author(s) 2015. This article is published with open access at Springerlink.com

\begin{abstract}
The aim of this study was to identify patients with methicillin-resistant Staphylococcus aureus (MRSA) bacteremia with low risk of infective endocarditis (IE) who might not require routine trans-esophageal echocardiography (TEE). We retrospectively evaluated 398 patients presenting with MRSA bacteremia for the presence of the following clinical criteria: intravenous drug abuse (IVDA), long-term catheter, prolonged bacteremia, intra-cardiac device, prosthetic valve, hemodialysis dependency, vertebral/nonvertebral osteomyelitis, cardio-structural abnormality. IE was diagnosed using the modified Duke criteria. Of 398 patients with MRSA bacteremia, $26.4 \%$ of cases were community-acquired, $56.3 \%$ were health-care-associated, and $17.3 \%$ were hospital-acquired. Of the group, 44 patients had definite IE, 119 had possible IE, and 235 had a rejected diagnosis. Out of 398 patients, 231 were evaluated with transthoracic echocardiography (TTE) or TEE.
\end{abstract}

Notation of prior abstract presentation: American Heart Association Scientific Meeting, 2012. "Simplified Criteria to Risk Stratify for the Mode of Echocardiography in Patients with MRSA Bacteremia at Low Risk of Infectious Endocarditis." Presentation \#15744. Los Angeles, California. Nov 2012.

P. Buitron de la Vega

pbuitro1@bu.edu

Division of Infectious Diseases, Henry Ford Health System, Detroit, MI, USA

2 Wayne State University School of Medicine, Detroit, MI, USA

3 Division of Internal Medicine, Ottawa Hospital, Ottawa, ON, Canada

4 Government Kilpauk Medical College Hospital, Chennai, India
All 44 patients with definite IE fulfilled at least one criterion (sensitivity $100 \%$ ). Finally, a receiver operator characteristic (ROC) curve was obtained to evaluate the total risk score of our proposed criteria as a predictor of the presence of IE, and this was compared to the ROC curve of a previously proposed criteria. The area under the ROC curve for our criteria was 0.710 , while the area under the ROC curve for the criteria previously proposed was $0.537(p<0.001)$. The $p$-value for comparing those 2 areas was less than 0.001 , indicating statistical significance. Patients with MRSA bacteremia without any of our proposed clinical criteria have very low risk of developing IE and may not require routine TEE.

\section{Abbreviations}

IE Infective endocarditis

ICD Implantable cardioverter-defibrillator
5 Heart and Vascular Institute, Henry Ford Health System, Detroit, MI, USA

6 Division of Internal Medicine, Boston Medical Center, 801 Massachusetts Ave Crosstown, 2nd Floor, Boston, MA 02118, USA

7 Boston University, Boston, MA, USA

8 Division of Cardiology, Department of Internal Medicine, Wake Forest University School of Medicine, Winston Salem, NC, USA

9 Department of Public Health Sciences, Henry Ford Health System, Detroit, MI, USA 
IVDA Intravenous drug abuse

PICC Peripherally inserted central catheter

MRSA Methicillin-resistant Staphylococcus aureus

ROC Receiver operator characteristic

SAB Staphylococcus aureus bacteremia

TEE Trans-esophageal echocardiography

TTE Trans-thoracic echocardiography

\section{Introduction}

Staphylococcus aureus bacteremia (SAB) is a leading cause of mortality and morbidity in both nosocomial and community settings [1-5]. It is the second most common cause of hospital bloodstream infections, and has become the leading cause of infective endocarditis (IE) in most parts of the world [6-11]. Despite recent advances in diagnosis and treatment, IE remains a serious and deadly disease $[8,12-16]$. Its 30-day all-cause mortality remains as high as $23.9 \%$ in left-sided IE and $11.8 \%$ in rightsided IE $[17,18]$. The classic peripheral stigmata of IE are often nonspecific or missing, particularly among patients in whom IE is the result of Staphylococcus aureus infection $[19,20]$. The high mortality of untreated IE, accompanied by a high prevalence of patients without clinical manifestations [8], emphasizes the importance of a diagnostic strategy sensitive enough for disease detection [21, 22]. The current European Society of Cardiology, the American College of Cardiology/American Heart Association guidelines for IE, and other recent studies recommend performing routine transthoracic echocardiography (TTE) in all patients with suspected IE [19, 23-25]. Furthermore, cost-effective calculations suggest that transesophageal echocardiography (TEE) should be done first in adults with suspected IE [19]. It has also been suggested that all patients with SAB should be considered as high risk for developing IE, and they should all undergo TTE/TEE evaluation [23, $24,26]$. Habib et al. suggested that a negative TTE in patients with SAB should be followed by TEE, due to the high clinical suspicion of IE in patients with $\mathrm{SAB}$ [27]. Whether all patients with SAB need a TEE is an unsettled issue. Recent literature suggests that further work is needed to identify a subgroup of patients with SAB that might only need TTE for their evaluations of IE [19]. It has been proposed that the absence of certain clinical characteristics can identify patients with SAB with low risk of IE that might not require TEE evaluation $[1,28]$. As such, the aim of our study was to identify patients with SAB with low risk of IE by using simplified prediction criteria that include common risk factors for IE.

\section{Methods}

\section{Hospital patients and settings}

We retrospectively identified all consecutive patients with methicillin-resistant Staphylococcus aureus (MRSA) bacteremia diagnosed at a large tertiary care center, Henry Ford Hospital in Detroit, Michigan from 2005 to 2009. Cases of MRSA bacteremia were identified from review of the records of the clinical microbiology laboratory. All patients $\geq 18$ years old with community-acquired, health-care-associated, or nosocomial MRSA who had $\geq 1$ blood culture positive for MRSA were included in the study. The Henry Ford Hospital Institutional Review Board approved the study protocol.

\section{Data acquisition}

Patients with MRSA bacteremia were evaluated for date, duration, epidemiologic source and number of positive blood cultures, source of infection, clinical signs of IE, presence of vascular events (emboli, hemorrhage), hemodialysis dependency, short-term catheter, implantable catheter, fistula or graft, diabetes, vertebral or non-vertebral osteomyelitis, prosthetic heart valve, intravenous drug use (IVDU), intra-cardiac device, cardio-structural abnormality, new conduction block, infective endocarditis diagnosis, type and timing of ultrasound studies, specialty of physician ordering the ultrasound.

\section{Definitions}

MRSA bacteremia was defined as $\geq 1$ positive blood culture, and it was considered (1) hospital-acquired if the blood culture was positive $\geq 48 \mathrm{~h}$ after admission and infection was not present or incubating at time of admission, (2) health-careassociated if infection was outpatient or within the first $48 \mathrm{~h}$ of hospitalization and the patient was hospitalized within the previous year, or (3) community-acquired if infection was outpatient or within the first $48 \mathrm{~h}$ of hospitalization and the patient was not hospitalized within the previous year [29]. The source of bacteremia was defined as the most likely source responsible for the first possible blood culture result on the basis of clinical signs, imaging, and microbiological findings. Prolonged bacteremia was considered "documented" if follow-up blood cultures yielded MRSA 2-4 days (at least $12 \mathrm{~h}$ after initial blood culture) after the first positive blood culture result. We selected this definition of prolonged bacteremia with the goal of making our criteria more sensitive to detect patients with lower risk of IE. If a patient did not have a follow-up blood culture, they were considered in the group of "possible" prolonged bacteremia. In our analysis, "possible" and "documented" bacteremia were considered as prolonged bacteremia. 
Short-term catheter was defined as any peripherally inserted central catheter (PICC), internal jugular vein catheter, subclavian vein catheter, radial arterial catheter, or femoral arterial catheter or arterial line. Implantable catheter was defined as any tunneled catheter or mediport. Intra-cardiac device was defined as any implantable cardioverter-defibrillator (ICD) or permanent pacemaker (PPM). Previous IE was defined as any prior episode of IE, whether related or not related to an infected intra-cardiac device. IE was defined as definite based on modified Duke criteria [30]. IE was excluded based on modified Duke criteria [30].

\section{Criteria set}

The criteria set was devised based on previous studies that have identified significant IE risk factors in a proposed clinical criteria including: prolonged bacteremia [31,32], permanent intra-cardiac device (e.g., a prosthetic heart valve, pacemaker, or cardioverter-defibrillator) [33, 34], hemodialysis dependency [35], spinal infection (e.g., vertebral osteomyelitis, epidural, subdural or intra-spinal empyema, or abscess) and nonvertebral osteomyelitis $[1,36,37]$. This criteria set was found to have a sensitivity of $97.5-100 \%$ and a negative predictive value of $92.2-100 \%$ [1]. One of its limitations is its applicability to other populations with different risk factor profiles. Other studies have also included risk factors such as intravenous drug use $[24,38]$, known native valve abnormality [24, $38]$, previous IE [24, 38], and presence of central venous access [38-40]. Taking into consideration these previously proposed risk factors and our population profile, we developed our modified criteria for predicting IE, including prolonged bacteremia, intravenous drug use, implantable catheter, intracardiac device, prosthetic valve, hemodialysis, osteomyelitis, previous infective endocarditis, and cardio-structural abnormality.

Echocardiography data: all echocardiograms (TTE and TEE) were performed by the American Society of Echocardiography Level 2 and 3 trained echocardiographers. A vegetation was defined as a definite oscillating mass, irregular in character, located on the atrial surface of the mitral and tricuspid valves or ventricular surface of the aortic or pulmonic valves. If there was suspicion of vegetation, abnormal leaflet thickening, abscess, or worsening of valvular regurgitation noted on TTE, a TEE was recommended as a follow-up study. If image quality on TTE was not sufficient to assess valves and mural endocardium, a TEE was also recommended for further evaluation.

\section{Data analysis}

The individual risk variables have been scored as 0 (no) or 1 (yes) and added together to create a total risk score. Then a cross-tabulation table was used to display the association of the total risk score with endocarditis status, with the CochranArmitage trend test used to evaluate the strength of that association. Furthermore, to evaluate the total risk score as a predictor of the presence of endocarditis, a receiver operating characteristic (ROC) curve was created, and the resulting area under the ROC curve was calculated. The optimal cut point from the total risk score was defined as the one containing the maximum sum of the sensitivity plus specificity for predicting endocarditis. Finally, logistic regression modeling was used to evaluate the multivariable association of endocarditis status with the individual risk variables and patient characteristics.

\section{Results}

A total of 398 patients with MRSA bacteremia were identified and included in the analysis. Of these, $59.8 \%$ were male, and the mean age was $58.6 \pm 17.1$ years: $105(26.4 \%)$ were community-acquired, $224(56.3 \%)$ were health-care-associated and $69(17.3 \%)$ were hospital-acquired. The median duration of the MRSA bacteremia was 4 ( 2 to 6 ) days. Of the patients in our cohort, $161(40.5 \%)$ had diabetes, 90 (22.6\%) were hemodialysis-dependent, 20 (5\%) had human immunodeficiency virus, $85(21.3 \%)$ were IVDU, and $19(4.7 \%)$ had a prosthetic valve. The demographic and other clinical features of the cohort are displayed in Table 1.

\section{Diagnosis of infective endocarditis}

The diagnosis of IE was classified as definite in 44 [11], possible in $119(29.9 \%)$ and rejected in $235(59 \%)$ based on modified Duke criteria. Of these, 14 (3.5\%) patients had tricuspid valve vegetation, 12 (3\%) mitral valve vegetation, and eight ( $2 \%)$ aortic valve vegetation. Of the total 398 patients, $241(60.5 \%)$ were evaluated with echocardiography (TTE or TEE). Of the 157 patients not evaluated with echocardiography, 116 (73.8 \%) were categorized as rejected infective endocarditis, based on the modified Duke criteria, nine (5.7\%) had a concurrent diagnosis of osteomyelitis/cellulitis, seven $(4.4 \%)$ expired during the initial hospital stay, two $(1.3 \%)$ left against medical advice before undergoing echocardiography, and one $(0.6 \%)$ was unable to proceed with TEE due to restricted cervical motion.

Of the total 241 patients evaluated with echocardiography, $114(47.3 \%)$ were evaluated with TTE only, 56 (23.2 \%) TEE only, and $71(29.5 \%)$ TTE followed by TEE. Of the 241 patients evaluated with echocardiography studies, 234 $(97.1 \%)$ had their initial study done within 14 days after the first positive blood culture result. Of the total 44 patients with definite IE diagnosis, 24 (54.5\%) were evaluated with both TTE and TEE, ten $(22.7 \%)$ were evaluated with TEE, and ten $(22.7 \%)$ were evaluated with TTE alone. 
Table 1 Characteristics of 398 patients with MRSA bacteremia

\begin{tabular}{|c|c|c|c|}
\hline Characteristics & $\begin{array}{l}\text { All patients } \\
(n=398)\end{array}$ & $\begin{array}{l}\text { Patients with IE } \\
(n=44)\end{array}$ & $\begin{array}{l}\text { Patients without } \\
\text { IE }(n=354)\end{array}$ \\
\hline Age & $58.6 \pm 17.1$ & $44 \pm 12.4$ & $59.7 \pm 17.3$ \\
\hline Male & $238(59.8 \%)$ & $24(54.5 \%)$ & $214(60.5 \%)$ \\
\hline Nursing home resident & $58(14.6 \%)$ & $2(4.5 \%)$ & $56(15.8 \%)$ \\
\hline \multicolumn{4}{|l|}{ MRSA bacteremia characteristics } \\
\hline Community & $105(26.4 \%)$ & $22(50 \%)$ & $83(23.4 \%)$ \\
\hline Health care-associated & $224(56.3 \%)$ & $18(40.9 \%)$ & $206(58.2 \%)$ \\
\hline Hospital-acquired & $69(17.3 \%)$ & $4(9.1 \%)$ & $65(18.4 \%)$ \\
\hline Duration of MRSA bacteremia (median) & 4.47 days & 6.86 days & 4.12 days \\
\hline \multicolumn{4}{|l|}{ Presumed source of infection } \\
\hline Graft infection & $10(2.5 \%)$ & $1(2.3 \%)$ & $9(2.5 \%)$ \\
\hline Short term catheter & $55(13.8 \%)$ & $0(0 \%)$ & $55(15.5 \%)$ \\
\hline Implantable catheter & $33(8.3 \%)$ & $3(6.8 \%)$ & $30(8.5 \%)$ \\
\hline Skin/wound & $132(33.2 \%)$ & $9(20.5 \%)$ & $123(34.7 \%)$ \\
\hline Intra-abdominal & $10(2.5 \%)$ & $1(2.3 \%)$ & $9(2.5 \%)$ \\
\hline Respiratory & $48(12.1 \%)$ & $9(20.5 \%)$ & $39(11 \%)$ \\
\hline Para-spinal abscess & $2(0.5 \%)$ & $1(2.3 \%)$ & $1(0.3 \%)$ \\
\hline Urinary tract & $36(9 \%)$ & $0(0 \%)$ & $36(10.3 \%)$ \\
\hline Other & $72(18.1 \%)$ & $20(45.5 \%)$ & $52(14.7 \%)$ \\
\hline \multicolumn{4}{|l|}{ Comorbidities } \\
\hline Diabetes & $161(40.5 \%)$ & $13(29.5 \%)$ & $148(41.8 \%)$ \\
\hline Hemodialysis & $90(22.6 \%)$ & $4(9.1 \%)$ & $86(24.3 \%)$ \\
\hline HIV & $20(5 \%)$ & $5(11.4 \%)$ & $15(4.2 \%)$ \\
\hline Nursing home resident & $58(14.6 \%)$ & $2(4.5 \%)$ & $56(15.8 \%)$ \\
\hline
\end{tabular}

\section{Prediction criteria for diagnosis of IE}

The significant clinical predictors for diagnosis of IE are provided in Table 2. We considered less than one positive variable as predicting low risk of IE. In total, 44 patients with documented IE fulfilled at least one criterion (sensitivity $100 \%$, negative predictive value $100 \%$, specificity $19.2 \%$, positive predictive value $13.3 \%$ ). Of the 44 patients with documented IE, 34 fulfilled at least two criteria (sensitivity $77.3 \%$, negative predictive value $95.2 \%$, specificity $55.6 \%$, and positive predictive value $17.8 \%$ ). Among the 68 patients that fulfilled less than one of the clinical predictors, $23(33.8 \%)$ were evaluated with TTE as the first imaging study, five (7.4\%) with TEE alone, one (1.5\%) underwent TEE followed by TTE, and 39 (57.5\%) did not have any imaging done. Of the 39 patients not evaluated with echocardiography, 33 (86.4\%) were categorized as rejected IE based on the modified Duke criteria, four (10.3\%) expired during the initial hospital stay, and in two cases $(5.1 \%)$, there was not enough information in the chart review to determine the reason for not evaluating with imaging.

A ROC curve was obtained using our criteria (Fig. 1) and was compared to the criteria proposed by Kaasch et al. [1] (Fig. 2), which included prolonged bacteremia, permanent intracardiac device, prosthetic heart valve, pacemaker, cardioverter- defibrillator, hemodialysis dependency, spinal infection, or non-vertebral osteomyelitis. The area under the ROC curve for our criteria was $0.710(p<0.001)$, while the area under the ROC curve for the simple criteria proposed by Kaasch et al. [1] was $0.537(p=0.296)$. When comparing the ROC curve areas to a reference area of 0.50 (the area that would be expected if no predictive ability existed), our criteria had significantly better ability to predict IE than Kaasch et al. [1] $(P<0.001)$.

\section{Association of the prediction criteria with mortality}

When applying our criteria to predict mortality, we determined that $19(27.9 \%)$ of the 68 patients with less than one of the proposed criteria expired within 30 days of their diagnosis of MRSA bacteremia, compared to $68(20.6 \%)$ of the 330 patients with one or more than one of the proposed criteria. We found a sensitivity of $78.2 \%$, a specificity of $15.8 \%$, a positive predictive value of $20.6 \%$ and a negative predictive value of $72.1 \%$ with a chi-square test $p$-value of 0.183 , demonstrating no statistically significant association of our proposed prediction criteria with mortality. When applying the criteria proposed by Kaasch et al. [1], we found that 19 $(22.9 \%)$ of the 83 patients with less than one of their proposed criteria died within 30 days of their diagnosis, compared to 68 
Table 2 Clinical prediction criteria associated with increased risk of IE in patients with MRSA bacteremia

\begin{tabular}{lllllrrr}
\hline Prediction criterion & All patients $(n=398)$ & Without IE $(n=354)$ & With IE $(n=44)$ & $P$-value & Odds ratio & $\begin{array}{l}\text { Odds ratio } \\
95 \% \text { confidence limits }\end{array}$ \\
\hline Prolonged bacteremia & $269(67.5 \%)$ & $232(65.5 \%)$ & $37(84.1 \%)$ & 0.167 & 2.131 & 0.729 \\
Intravenous drug use & $85(21.3 \%)$ & $53(15 \%)$ & $32(72.7 \%)$ & $<0.001 *$ & 14.951 & 5.356 & 41.734 \\
Implantable catheter & $60(15 \%)$ & $57(16.1 \%)$ & $3(6.8 \%)$ & 0.387 & 3.441 & 0.209 & 56.657 \\
Intracardiac device & $19(4.7 \%)$ & $18(5.1 \%)$ & $1(2.3 \%)$ & 0.384 & 0.280 & 0.016 & 4.917 \\
Prosthetic valve & $19(4.7 \%)$ & $7(2 \%)$ & $6(13.6 \%)$ & $0.002 *$ & 13.086 & 2.529 & 67.704 \\
Hemodialysis & $90(22.6 \%)$ & $86(24.3 \%)$ & $4(9.1 \%)$ & 0.122 & 0.068 & 0.002 & 2.040 \\
Osteomyelitis & $52(13 \%)$ & $46(13 \%)$ & $6(13.6 \%)$ & 0.067 & 0.287 & 0.076 & 1.089 \\
Previous IE & $19(4.7 \% \%)$ & $10(2.8 \%)$ & $9(20.5 \%)$ & 0.117 & 2.849 & 0.769 & 10.554 \\
Cardio-structural abnormality & $13(3.2 \%)$ & $9(2.5 \%)$ & $4(9.1 \%)$ & $0.035 *$ & 6.095 & 1.132 & 32.833 \\
\hline
\end{tabular}

* Statistically significant, $P<0.05$

( $27.5 \%$ ) of the 247 patients with one or more than one of the proposed criteria. We found a sensitivity of $78.2 \%$, specificity of $20.6 \%$, positive predictive value of $21.6 \%$ and a negative predictive value of 77.1 with a chi-square test $p$-value of 0.798 , also showing no statistically significant association of their proposed prediction criteria with mortality.

\section{Discussion}

Our study accurately demonstrated that our simplified criteria to risk stratify $\mathrm{SAB}$ patients effectively predicted patients with
MRSA bacteremia with very low risk of developing IE. Previous analysis by Kaasch et al. also demonstrated a high sensitivity to diagnose IE by using simplified criteria [1], although when the same criteria set was applied to our cohort population, the area under the curve was smaller than our proposed criteria that also included implantable catheter, IVDA, cardio-structural abnormality and previous IE. These four extra criteria added in our study represent important risk factors in an inner-city population with high prevalence of IVDU and implantable catheters.

In this retrospective analysis of patients with MRSA bacteremia, $44(11 \%)$ were diagnosed with infective endocarditis.
Fig. 1 ROC curve for our proposed clinical prediction criteria set

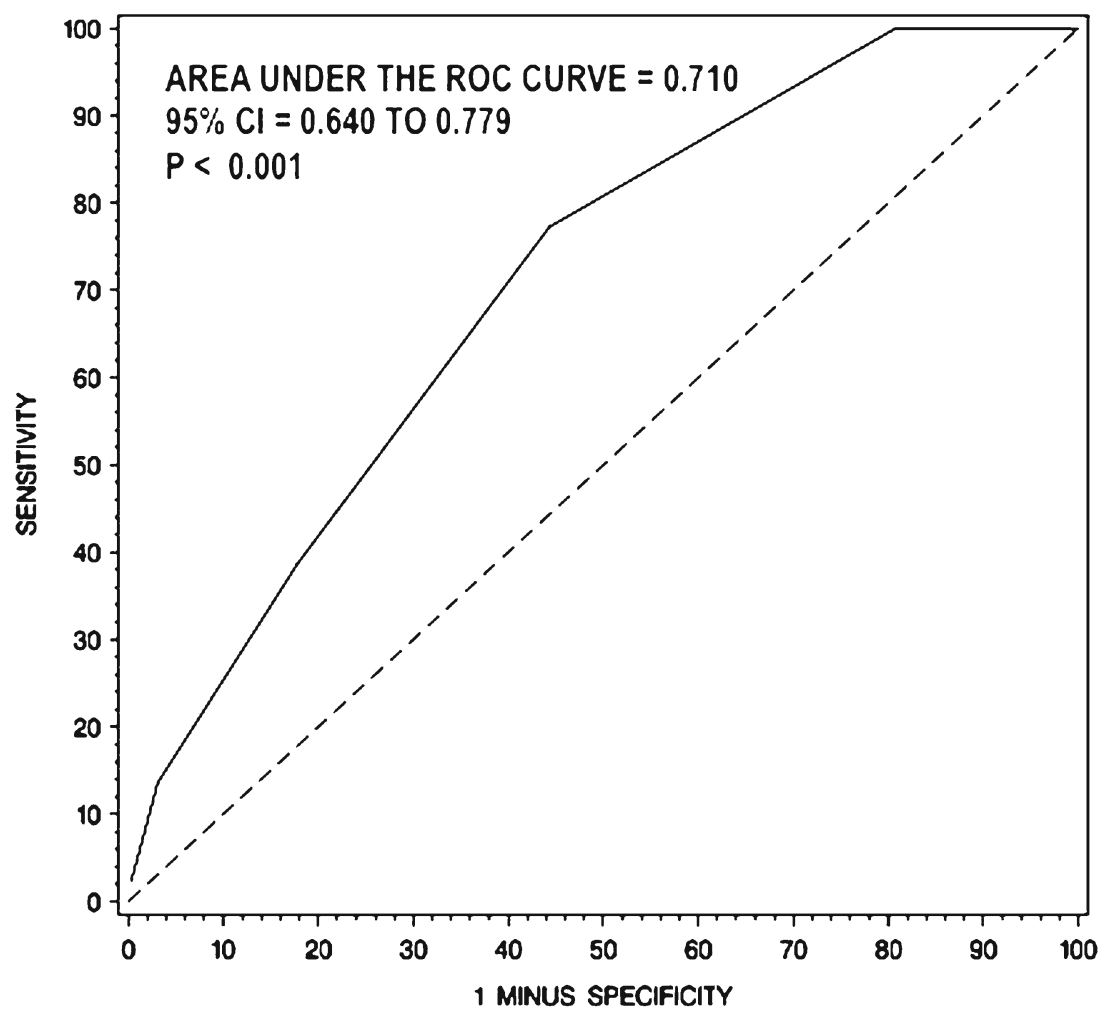


Fig. 2 ROC curve for clinical prediction criteria set proposed by Kaasch et al. [1]

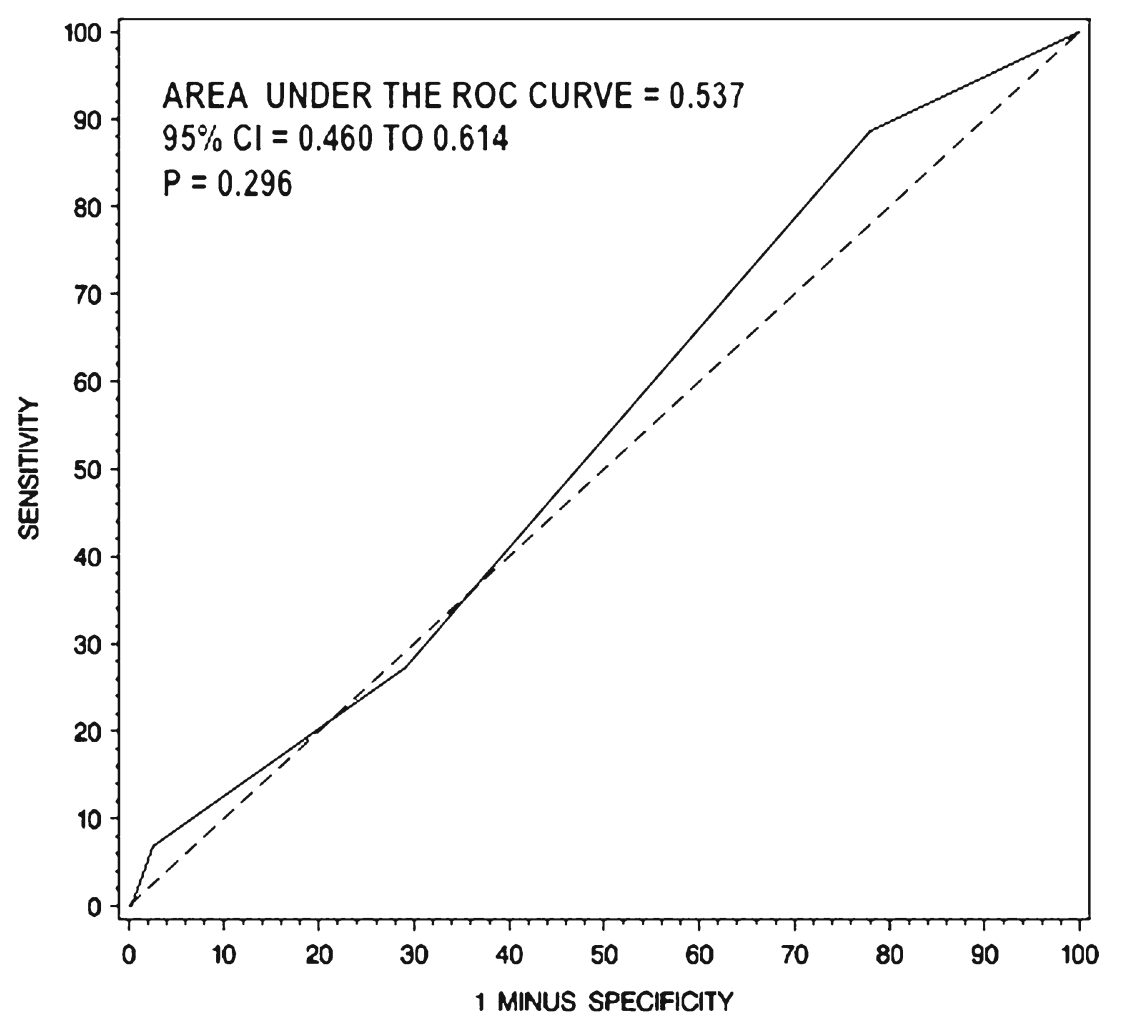

This prevalence of endocarditis in SAB is similar to previous studies that have reported values between 5 and $17 \%$ [1, 41-43,] and other studies have reported values up to $32 \%$ $[24,44,45]$. The 44 patients with IE had a 30-day overall mortality of $25 \%$ that is comparable to other reported studies ranging from 13 to $31 \%$ mortality $[2,41]$.

It is interesting to point out the lack of association of our criteria with mortality. One of the possible explanations of this result could be the fact that patients with more risk factors are provided with advanced treatment protocols and early empirical antimicrobial therapy. As has been previously reported, inadequacy of empirical antimicrobial therapy in these patients is an important risk factor for mortality [46]. It has also been reported that the APACHE II score has been associated with mortality in patients with MRSA bacteremia [47]. Most of the included variables in the APACHE II are not included in our prediction criteria, as they mainly measure hemodynamic values of the patients that are not necessary associated with IE.

\section{Clinical implications}

Although the current European Society of Cardiology, the ACC/AHA guidelines and other recent studies recommend performing routine echocardiography in all patients with SAB [19, 23, 24], only $241(60.5 \%)$ of the cases in our study complied with this recommendation, being one of the highest reported [48]. Other studies have shown compliance results ranging from 39.9 to $73 \%[1,24,49,50]$. Even in a cohort of patients with $\mathrm{SAB}$ presenting with intra-cardiac device and higher risk of endocarditis, echocardiography was obtained in only $77 \%$ of cases [51]. An accurate definition of low probability of IE in patients with SAB is still lacking, and is necessary to provide better guidance of how to use echocardiography. Our criteria could potentially be used as a tool to identify patients with MRSA bacteremia at low risk of endocarditis, in order to guide the mode of echocardiography. In patients with one or more of the proposed criteria, the risk of developing IE is higher, and TEE should be considered in their evaluation. In patients with less than one of the proposed criteria, their risk of IE is low and TEE might not be needed for their evaluation. In our cohort, TEE results did not change or add to the management in any of the low-risk patients. It could be argued that in patients with community-acquired MRSA and very low risk of $\mathrm{IE}$, the real duration of bacteremia is unknown, and as such, considering ordering a TTE should not be discouraged. While patients with less than one of the proposed criteria are at low risk of IE, they are still at very high risk of poor outcomes. Absence of IE does not imply absence of complicated disease in patients with MRSA bacteremia. It will be important to validate these criteria prospectively and follow the subjects in the long term in order to include missed cases of endocarditis.

\section{Limitations}

As our analysis was retrospective, there are several inherent limitations in this type of study design. Adequately identifying missed cases of IE was not possible, as there was no follow-up 
arranged. With the goal of capturing patients with missed IE, we performed a second chart review of medical records that corresponded to 6 months after MRSA was initially diagnosed. Even though we did not find any information supporting missed IE in patients with none of our prediction criteria, the review was still limited by the fact that we did not capture the actual cause of death, so some patients could have followed up at another institution or died before having a complete evaluation for IE. It is important to mention that source control was at discretion of the clinicians caring for the patient; as a retrospective chart review, this information was not consistently available. Additionally, not all patients in our cohort underwent TTE or TEE evaluation, limiting the diagnostic power of Duke criteria due to the fact that IE is not easily recognized by physical examination. This also limited our ability to classify patients as rejected IE. We performed a second chart review of all 116 patients that were classified as rejected IE, with the goal of identifying the reasons why no imaging was done. All of these 116 patients either had a firm alternate diagnosis for the manifestations of IE, the manifestations of infection resolved within 4 days, or they did not meet criteria for possible IE. The use of Duke criteria in these patients continues to be limited due to the fact that we did not have echocardiography evaluations or pathologic evidence at surgery or autopsy from any of them. As such, the actual number of IE cases may have been underestimated.

\section{Conclusion}

In conclusion, patients with MRSA bacteremia and at least one of our proposed clinical criteria have an inherently higher risk of IE, and TEE should be considered for their evaluation. Patients with less than one of our proposed criteria have very low risk of developing IE and might not need TEE for their evaluation. It is important to mention that in patients with less than one of our proposed criteria, physicians should not be discouraged from performing TTE in their evaluation, as such patients are still considered at risk of developing IE.

Acknowledgments Dr. Pablo Buitron is the guarantor of the content of the manuscript, including the data and analysis. There are no financial or non-financial disclosures associated with the work of this article.

\section{Compliance with ethical standards}

Conflict of interest None for all authors.

Open Access This article is distributed under the terms of the Creative Commons Attribution 4.0 International License (http:// creativecommons.org/licenses/by/4.0/), which permits unrestricted use, distribution, and reproduction in any medium, provided you give appropriate credit to the original author(s) and the source, provide a link to the Creative Commons license, and indicate if changes were made.

\section{References}

1. Kaasch AJ, Fowler VG Jr, Rieg S et al (2011) Use of a simple criteria set for guiding echocardiography in nosocomial Staphylococcus aureus bacteremia. Clin Infect Dis 53:1-9

2. Bassetti M, Trecarichi EM, Mesini A et al (2011) Risk factors and mortality of healthcare-associated and community-acquired Staphylococcus aureus bacteraemia. Clin Microbiol Infect 18: 862-869

3. Benfield T, Espersen F, Frimodt-Moller N et al (2007) Increasing incidence but decreasing in-hospital mortality of adult Staphylococcus aureus bacteraemia between 1981 and 2000. Clin Microbiol Infect 13:257-263

4. El-Ahdab F, Benjamin DK Jr, Wang A et al (2005) Risk of endocarditis among patients with prosthetic valves and Staphylococcus aureus bacteremia. Am J Med 118:225-229

5. Rieg S, Peyerl-Hoffmann G, de With K, Theilacker C et al (2009) Mortality of S. aureus bacteremia and infectious diseases specialist consultation - a study of 521 patients in Germany. J Infect 59:232 239

6. Hoen B, Alla F, Selton-Suty C et al (2002) Changing profile of infective endocarditis: results of a 1-year survey in France. JAMA 288:75-81

7. Cabell CH, Jollis JG, Peterson GE et al (2002) Changing patient characteristics and the effect on mortality in endocarditis. Arch Intern Med 162:90-94

8. Murdoch DR, Corey GR, Hoen B et al (2009) Clinical presentation, etiology, and outcome of infective endocarditis in the 21st century: the International Collaboration on Endocarditis-Prospective Cohort Study. Arch Intern Med 169:463-473

9. Federspiel JJ, Stearns SC, Peppercorn AF, Chu VH, Fowler VG Jr (2012) Increasing US rates of endocarditis with Staphylococcus aureus: 1999-2008. Arch Intern Med 172:363-365

10. Kern WV (2010) Management of Staphylococcus aureus bacteremia and endocarditis: progresses and challenges. Curr Opin Infect Dis 23:346-358

11. Wisplinghoff H, Bischoff T, Tallent SM, Seifert H, Wenzel RP, Edmond MB (2004) Nosocomial bloodstream infections in US hospitals: analysis of 24,179 cases from a prospective nationwide surveillance study. Clin Infect Dis 39:309-317

12. Benito N, Miro JM, de Lazzari E et al (2009) Health care-associated native valve endocarditis: importance of non-nosocomial acquisition. Ann Intern Med 150:586-594

13. Hill EE, Herijgers P, Claus P, Vanderschueren S, Herregods MC, Peetermans WE (2007) Infective endocarditis: changing epidemiology and predictors of 6-month mortality: a prospective cohort study. Eur Heart J 28:196-203

14. Fowler VG Jr, Miro JM, Hoen B et al (2005) Staphylococcus aureus endocarditis: a consequence of medical progress. JAMA 293:30123021

15. Cosgrove SE, Sakoulas G, Perencevich EN, Schwaber MJ, Karchmer AW, Carmeli Y (2003) Comparison of mortality associated with methicillin-resistant and methicillin-susceptible Staphylococcus aureus bacteremia: a meta-analysis. Clin Infect Dis 36:53-59

16. Kaasch AJ, Barlow G, Edgeworth JD et al (2014) Staphylococcus aureus bloodstream infection: a pooled analysis of five prospective, observational studies. J Infect 68:242-251

17. Thuny F, Di Salvo G, Belliard O et al (2005) Risk of embolism and death in infective endocarditis: prognostic value of echocardiography: a prospective multicenter study. Circulation 112:69-75

18. Turnidge JD, Kotsanas D, Munckhof W et al (2009) Staphylococcus aureus bacteraemia: a major cause of mortality in Australia and New Zealand. Med J Aust 191:368-373 
19. Baddour LM, Wilson WR, Bayer AS et al (2015) Infective endocarditis in adults: diagnosis, antimicrobial therapy, and management of complications: a scientific statement for healthcare professionals from the American Heart Association. Circulation 132(15):14351486

20. Fowler VG Jr, Li J, Corey GR et al (1997) Role of echocardiography in evaluation of patients with Staphylococcus aureus bacteremia: experience in 103 patients. J Am Coll Cardiol 30:1072-1078

21. Roder BL, Wandall DA, Frimodt-Moller N, Espersen F, Skinhoj P, Rosdahl VT (1999) Clinical features of Staphylococcus aureus endocarditis: a 10-year experience in Denmark. Arch Intern Med 159: 462-469

22. Casella F, Rana B, Casazza G et al (2009) The potential impact of contemporary transthoracic echocardiography on the management of patients with native valve endocarditis: a comparison with transesophageal echocardiography. Echocardiography 26:900-906

23. Bonow RO, Carabello BA, Chatterjee K et al (2008) 2008 Focused update incorporated into the ACC/AHA 2006 guidelines for the management of patients with valvular heart disease: a report of the American College of Cardiology/American Heart Association Task Force on Practice Guidelines (Writing Committee to Revise the 1998 Guidelines for the Management of Patients With Valvular Heart Disease): endorsed by the Society of Cardiovascular Anesthesiologists, Society for Cardiovascular Angiography and Interventions, and Society of Thoracic Surgeons. Circulation 118: e523-e661

24. Rasmussen RV, Host U, Arpi M et al (2011) Prevalence of infective endocarditis in patients with Staphylococcus aureus bacteraemia: the value of screening with echocardiography. Eur J Echocardiogr 12:414-420

25. Chu VH, Bayer AS (2007) Use of echocardiography in the diagnosis and management of infective endocarditis. Curr Infect Dis Rep 9:283-290

26. Van Hal SJ, Mathur G, Kelly J, Aronis C, Cranney GB, Jones PD (2005) The role of transthoracic echocardiography in excluding left sided infective endocarditis in Staphylococcus aureus bacteraemia. J Infect 51:218-221

27. Habib G, Hoen B, Tornos P et al (2009) Guidelines on the prevention, diagnosis, and treatment of infective endocarditis (new version 2009): the Task Force on the Prevention, Diagnosis, and Treatment of Infective Endocarditis of the European Society of Cardiology (ESC). Endorsed by the European Society of Clinical Microbiology and Infectious Diseases (ESCMID) and the International Society of Chemotherapy (ISC) for Infection and Cancer. Eur Heart J 30:2369-2413

28. Showler A, Burry L, Bai AD et al (2015) Use of transthoracic echocardiography in the management of low-risk staphylococcus aureus bacteremia: results from a retrospective multicenter cohort study. J Am Coll Cardiol Img 8:924-931

29. Garner JS, Jarvis WR, Emori TG, Horan TC, Hughes JM (1988) CDC definitions for nosocomial infections, 1988. Am J Infect Control 16:128-140

30. Li JS, Sexton DJ, Mick N et al (2000) Proposed modifications to the Duke criteria for the diagnosis of infective endocarditis. Clin Infect Dis 30:633-638

31. Neuner EA, Casabar E, Reichley R, McKinnon PS (2010) Clinical, microbiologic, and genetic determinants of persistent methicillinresistant Staphylococcus aureus bacteremia. Diagn Microbiol Infect Dis 67:228-233

32. Fowler VG Jr, Justice A, Moore C et al (2005) Risk factors for hematogenous complications of intravascular catheter-associated Staphylococcus aureus bacteremia. Clin Infect Dis 40:695-703

33. Hill EE, Vanderschueren S, Verhaegen J et al (2007) Risk factors for infective endocarditis and outcome of patients with Staphylococcus aureus bacteremia. Mayo Clin Proc 82:1165-1169
34. Uslan DZ, Sohail MR, St Sauver JL et al (2007) Permanent pacemaker and implantable cardioverter defibrillator infection: a population-based study. Arch Intern Med 167:669-675

35. Robinson DL, Fowler VG, Sexton DJ, Corey RG, Conlon PJ (1997) Bacterial endocarditis in hemodialysis patients. Am J Kidney Dis 30:521-524

36. Pigrau C, Almirante B, Flores X et al (2005) Spontaneous pyogenic vertebral osteomyelitis and endocarditis: incidence, risk factors, and outcome. Am J Med 118:1287

37. Jensen AG, Espersen F, Skinhoj P, Rosdahl VT, Frimodt-Moller N (1997) Increasing frequency of vertebral osteomyelitis following Staphylococcus aureus bacteraemia in Denmark 1980-1990. J Infect 34:113-118

38. Knudsen JB, Fuursted K, Petersen E et al (2011) Failure of clinical features of low probability endocarditis. The early echo remains essential. Scand Cardiovasc J 45:133-138

39. Crowley AL, Peterson GE, Benjamin DK Jr et al (2008) Venous thrombosis in patients with short- and long-term central venous catheter-associated Staphylococcus aureus bacteremia. Crit Care Med 36:385-390

40. Miro JM, Anguera I, Cabell CH et al (2005) Staphylococcus aureus native valve infective endocarditis: report of 566 episodes from the International Collaboration on Endocarditis Merged Database. Clin Infect Dis 41:507-514

41. Chang FY, MacDonald BB, Peacock JE Jr, Musher DM, Triplett P, Mylotte JM et al (2003) A prospective multicenter study of Staphylococcus aureus bacteremia: incidence of endocarditis, risk factors for mortality, and clinical impact of methicillin resistance. Medicine 82:322-332

42. Das I, O'Connell N, Lambert P (2007) Epidemiology, clinical and laboratory characteristics of Staphylococcus aureus bacteraemia in a university hospital in UK. J Hosp Infect 65:117-123

43. Fowler VG Jr, Olsen MK, Corey GR et al (2003) Clinical identifiers of complicated Staphylococcus aureus bacteremia. Arch Intern Med 163:2066-2072

44. Abraham J, Mansour C, Veledar E, Khan B, Lerakis S (2004) Staphylococcus aureus bacteremia and endocarditis: the Grady Memorial Hospital experience with methicillin-sensitive $\mathrm{S}$ aureus and methicillin-resistant S aureus bacteremia. Am Heart J 147:536-539

45. Vos FJ, Bleeker-Rovers CP, Sturm PD et al (2011) Endocarditis: effects of routine echocardiography during Gram-positive bacteraemia. Neth J Med 69:335-340

46. Chen R, Yan ZQ, Feng D, Luo YP, Wang LL, Shen DX (2012) Nosocomial bloodstream infection in patients caused by Staphylococcus aureus: drug susceptibility, outcome, and risk factors for hospital mortality. Chin Med J 125:226-229

47. Stevens V, Lodise TP, Tsuji B et al (2012) The utility of acute physiology and chronic health evaluation II scores for prediction of mortality among intensive care unit (ICU) and non-ICU patients with methicillin-resistant Staphylococcus aureus bacteremia. Infect Control Hosp Epidemiol 33:558-564

48. Naber CK, Baddour LM, Giamarellos-Bourboulis EJ et al (2009) Clinical consensus conference: survey on Gram-positive bloodstream infections with a focus on Staphylococcus aureus. Clin Infect Dis 48(Suppl 4):S260-S270

49. Honda H, Krauss MJ, Jones JC, Olsen MA, Warren DK (2010) The value of infectious diseases consultation in Staphylococcus aureus bacteremia. Am J Med 123:631-637

50. Nagao M, linuma Y, Saito T et al (2009) Close cooperation between infectious disease physicians and attending physicians results in better outcomes for patients with Staphylococcus aureus bacteraemia. Clin Microbiol Infect 16:1783

51. Uslan DZ, Dowsley TF, Sohail MR et al (2010) Cardiovascular implantable electronic device infection in patients with Staphylococcus aureus bacteremia. Pacing Clin Electrophysiol 33: $407-413$ 